\title{
Principios de marketing y competitividad como estrategias claves para el desarrollo de las MIPYMES en la provincia de El Oro
}

\section{Principles of marketing and competitiveness as key strategies for the development of MSMEs in the province of El Oro}

Ana Vanessa Maldonado Córdova

Universidad Metropolitana, Ecuador

Universidad de Palermo, Argentina

Nancy María del Carmen Franco Gómez

Universidad Metropolitana, Ecuador

Autor para correspondencia: amaldonado@umet.edu.ec, mfranco@umet.edu.ec

Fecha de recepción: 02 de agosto de 2018 - Fecha de aceptación: 15 octubre de 2018

Resumen: El marketing y la competitividad son dos términos claves dentro del desarrollo de todo tipo de economía, por tal razón es importante realizar una asociación entre ambos, para lo cual se realizó la aplicación de un cuestionario a 40 empresas de la ciudad de Machala y se pudieron obtener resultados muy interesantes con respecto a estas dos áreas de estudio. Por un lado el presente estudio se realizó en empresas de varios sectores para poder tener una perspectiva un poco más general acerca de las condiciones actuales del mercado, así también como la ubicación de las organizaciones dentro de las principales ciudades de la provincia de El Oro. Otro de los aspectos que se tomó en consideración es el hecho de conocer si las empresas cuentan o no con una persona profesional encargada del área de marketing, ya que de esta manera se puede garantizar una mejor aplicación de los conceptos en esta área que necesita tener presente una organización para poder destacar con mayor fuerza. Es importante enfatizar que el marketing muchas veces es considerado como un mero gasto de publicidad dentro de las organizaciones, sobre todo si estas son de tipo pequeñas o medianas, sin embargo con el paso del tiempo se ha ido cambiando ese tipo de perspectiva y se ha podido identificar el gran aporte que genera a las empresas y sus repercusiones en el mejoramiento de la competitividad de las mismas, por ende el reflejo de la presente investigación que se presentará a continuación permitirá conocer de una manera aplicada la relación existente entre el marketing y la competitividad enfocada específicamente en ciertas ciudades de la provincia de El Oro y los diferentes estándares o estrategias que las empresas realizan.

Palabras Claves: marketing; competitividad; estrategia; desarrollo de negocios; empresas Abstract: Marketing and competitiveness are two key terms in the development of any type of economy, for this reason it is important to make an association between both, for which the application of a questionnaire was applied to 40 companies in the city of Machala. get very interesting results with respect to these two areas of study. On the one hand, the present study was carried out in companies from various sectors in order to have a somewhat more general perspective about the current market conditions, as well as the location of the organizations within the main cities of the province of El Oro. Another aspect that was taken into consideration is the fact of knowing whether or not companies have a professional person in charge of the marketing 
area, since this way you can guarantee a better application of the concepts in this area that you need to have present an organization to be able to stand out with greater force. It is important to emphasize that marketing is often considered as a mere expenditure of advertising within organizations, especially if they are small or medium-sized, however with the passage of time that type of perspective has been changed and was able to identify the great contribution that it generates to the companies and their repercussions in the improvement of the competitiveness of the same ones, therefore the reflection of the present investigation that will be presented next will allow to know in an applied way the existing relation between the marketing and the competitiveness specifically focused on certain cities in the province of El Oro and the different standards or strategies that companies perform.

Key Words: marketing; competitiveness; strategy; business development; companies

\section{Introducción}

Dentro de un mundo en constantes cambios, estar a la vanguardia de los avances: científicos, tecnológicos, niveles de demanda de productos de alta calidad; los cuales han transformado los patrones de producción. Y sostener una ventaja competitiva es casi de vida o muerte para las empresas grandes y más aún para las pequeñas empresas que recién están inician en el mundo empresarial.

Las pequeñas y medianas empresas de este siglo tienen grandes retos, ya que actualmente las empresas sin importar su tamaño se encuentran en constante renovación. Es por eso que se a conseja a los dueños o gerentes propietarios de las pequeñas y medianas empresas realizar constantemente auto análisis para tener una cultura de prevención ante los constantes cambios.

Es por eso que el presente estudio pretende analizar la importancia de contar con personal o un área de marketing especializado.

En primera instancia el estudio se centró en un análisis bibliográfico, para analizar los principales conceptos del marketing sus estrategias, luego se formuló un instrumento para recabar informar de las pequeñas y medianas empresas de la Provincia de El Oro.

La relevancia de esta investigación, radica en describir como las mipymes de la provincia de El Oro desarrollan sus actividades y como están deben adaptarse a una competencia más competitiva.

\section{Fundamentación teórica}

\section{Marketing}

Para comprender más detalladamente la importancia del marketing se presenta la interpretación de tres autores renombrado en el área, (Kotler, 1995) destaca que el marketing "es un proceso administrativo y social, mediante los futuros compradores obtienen lo que necesitan", el marketing ya no es se lo puede ver a corto plazo ni como un gasto, el marketing debe ser visto como una inversión (Srivastava, 1998). Sin embargo para (AMA, 2004) señala que el marketing es una función de la organización y un conjunto de procesos para la creación, comunicación, y 
entrega de valor para gestionar arduamente las relaciones con los clientes, donde exista un bien común cliente-empresa

\section{Marketing estratégico}

El marketing estratégico es la ciencia donde se reconoce las necesidades y deseos de los clientes actuales y potenciales y con esto poder obtener ventajas competitivas a mediano y largo plazo. Las estrategias basadas en competidores y especialmente en el cliente tienden a ser coherentes con y sostenibles a largo plazo (Wind, 1983).

\section{Estrategia}

Las empresas pueden valerse de tres tipos de estrategias de marketing, (McClymont, 2003) propone en primera instancia la estrategia pasiva: que determina realizar las tácticas comúnmente usadas en el marketing y esperar que los clientes respondan a los mismos; la estrategia activa: las empresas con más tiempo en el mercado y basados en su experiencia satisfacen a sus clientes de acuerdo a características propias de cada uno; y por último la estrategia científica: cuando se basan en modelos matemáticos para seleccionar al cliente, prospectando y proyectando los patrones de comportamiento del mercado meta.

\section{Desarrollo de negocio}

\section{Competitividad}

Según (Kester \& Luehrman, 1989), la competitividad es la capacidad de generar bienes y servicios creando una valor agregado para diferenciarse de la competencia, mientras que (Villareal, 2006) destaca que la competitividad está más allá del simple hecho de la productividad, representa un proceso fortalecido en generar fortalezas y capacidades productivas y organizacionales, crean una capacidad de adaptarse a los cambios tanto sociales como económicos que presenta la sociedad o los mercados metas, requiere que las empresas desarrollen nuevos comportamientos y procesos dentro de la organización, a todos estos cambio se corre el riesgo de incurrir en costos y limitar la búsqueda de información para incursionar en nuevos mercados (George, 2002)

\section{Estrategia competitiva}

Para (Munuera, 2007), una empresa posee una estratégica competitiva cuando esta dispone de características y atributos que son superiores a los de su competencia y es sostenible en el tiempo, estos atributos contribuyen a que el consumidor perciba este valor manifestado en la superioridad en costos, comercialización o en la calidad del producto en si. (Wensley, 2010).

En la figura 1 se representa como las estrategias pueden desarrollar una ventaja competitiva. 


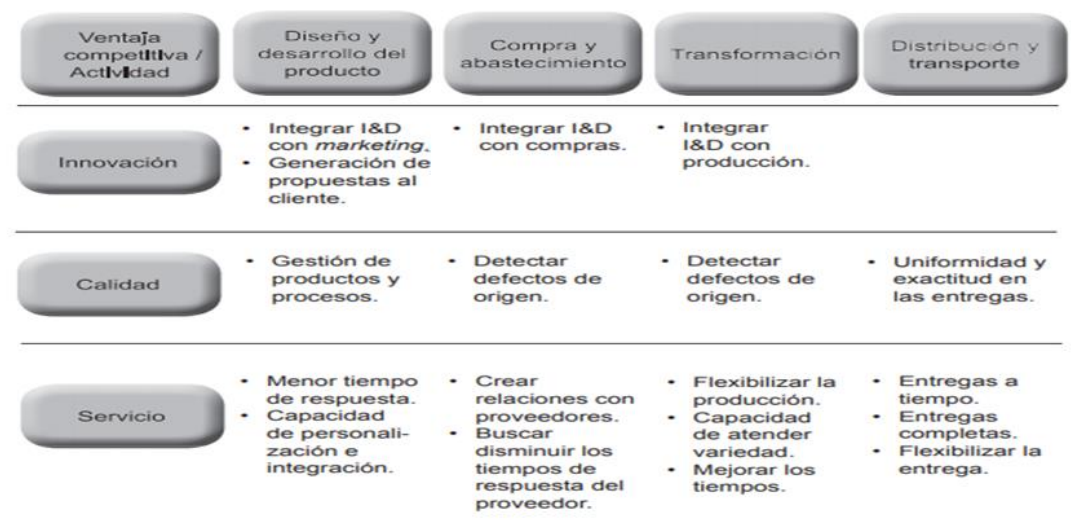

Figura: 1

Autor: (Chirinos Cuadros \& Rosado Samaniego, 2018)

\section{Pequeñas empresas}

Existe una gran diferencia entre las empresas grandes y pequeñas o medianas, dadas que las segundas son menos burocratitas y flexibles. El gerente propietario es quien toma las decisiones a nivel general (Carson \& Cromie, 2010); es por esto que el crecimiento de las pequeñas empresas es tan variable ya que según (Poutziouris, 2003), son impulsadas por la interacción entre los recursos y la motivación del propietario; el perfil del negocio: tamaño, antigüedad; y la planificación estratégica: recursos, posicionamiento del mercado, desarrollo.

\section{Pyme de éxito}

El éxito de una empresa depende de su capacidad para renovarse, mantener y aumentar su posicionamiento en el mercado (Kester \& Luehrman, 1989), mientras que (Ramos, 2001) sostiene que un país depende de la capacidad de las empresas para renovarse, desarrollar factores de éxito para el crecimiento económico a mediano y largo plazo.

Desde el punto de vista de (Donrrosoro, et al., 2001), los activos tecnológicos que posee la empresa son de gran importancia ya que son de gran importancia a la hora de producir bienes y servicios, es por esto que la adquisición de activos tecnológicos determina en gran parte su potencial a la hora de tener mayor competitividad. No se puede dejar de lado la capacidad de adaptación a consumidores cada vez más cambiantes y dinámicos (Donrrosoro, et al., 2001)

\section{Pymes}

Según datos (Inec, 2012) 2012, el 89,6\% pertenece a las microempresas, el 8,2\% a la pequeña empresa, el 1,7\% a la mediana empresa y solo el $0,5 \%$ a las grandes empresas a nivel nacional.

Predominan las empresas dedicadas el comercio con un $39 \%$ seguido de la agricultura y ganadería con un $14,7 \%$, almacenamiento y transporte con $8,4 \%$; manufacturas $7,4 \%$ y alojamiento y bebidas con el 7,3\%. 
Dado las distintas variables las MiPymes pueden tener un mismo comportamiento frente a la creación de un departamento de marketing o la contratación de un entendido en la materia. Y dada la poca información que existe sobre el mismo se importante el estudio de las mismas como un todo

\section{Metodología}

\section{Diseño}

Se decidió llevar a cabo un primer estudio sobre una base de 559 Empresas de pequeñas y medianas en la provincia de El Oro, siendo Machala como ciudad capital, la ciudad con mayor número de empresas. Se llevó a cabo 41 Encuestas personales realizadas a los gerentes o encargado de cada una de las empresas.

La investigación desarrollada es de tipo descriptiva, para lo cual se realizó un estudio generalizado acerca de las variables tratadas en el trabajo de investigación, posteriormente se procedió a realizar una descripción detallada de los diferentes subtemas más relevantes para el objeto de estudio.

\begin{tabular}{l} 
Ficha técnica \\
\hline Procedimiento Metodológico: Encuestas personales a través de cuestionarios totalmente estructurados \\
Población de estudio: 559 empresas \\
Ámbito: Provincia de El Oro \\
Lugar de contacto: Sede de las empresas \\
Muestra definitiva: 41 encuestas que corresponde al 7,33\% del total de las empresas \\
Fecha trabajo de campo: 2 a 20 de abril del 2018
\end{tabular}

\section{Instrumentos}

La información fue recopilada a través de diferentes medios, por un lado una de las principales como lo es el internet a través de la plataforma de Google Académico, así también se consultaron artículos académicos publicados en revistas que se encontraron en bases de datos tales como Science Direct, Scopus, Ebsco y Scielo; y a través de encuestas como se explica anteriormente.

\section{Procedimientos}

La revisión bibliográfica realizada arrojó como resultado 122 documentos consultados, de los cuales fueron utilizados 18 considerados como los más relevantes para el objeto de la información.

Para poder seleccionar los artículos se tomaron en consideración el resumen y las conclusiones del mismo, de esta manera ambas partes sirvieron como referencia de exclusión o inclusión respectivamente. Por otro lado, la información fue organizada en aquellos artículos que hacían referencia a las estrategias de la competitividad y por otro lado aquellos documentos que tenían contenido basado en el desarrollo sostenible.

\section{Descripción de resultados}


Para poder realizar la descripción de los resultados es importante analizar una de una las preguntas realizadas.

Capacidad Financiera: esta pregunta está compuesta 1 la empresa destina presuesto para el departamento de marketing y 2 si no lo destina.

Capacidades de Marketing: Se les pregunto a los encargados o gerentes que actividades realiza para un mejor desempeño competitivo; 1 analizar el entorno competitivo, 2 ofrecer un nuevo servicio, 3 investigar los cambios en los hábitos de consumo, 4 Reducción significativa de los costos para ofrecer un precio más competitivo.

Formación: Cual es el nivel de formación que actualmente tiene el responsable del área de mercadeo, 1 técnico, 2 tecnológico, 3 profesional, 4 postgrado 5 ninguno.

Realizando un análisis general de las empresas según resultados de la encuesta analizamos que el $83 \%$ de las MiPymes posee un responsable del área de mercado, con esto acotamos que cada vez están interesada en el reconocimiento de mercadeo ya que el $71 \%$ de las mismas destinan presupuesto para el área.

Entre las principales actividades que se realiza resalta el analizar el entorno y ofrecer un nuevo servicio, acotamos que estamos dos variables van de la mano, ya que al analizar nuestra competencia podemos realizar un análisis y ofrecer servicio que nuestra competencia no posee; sin embargo, solo el $50 \%$ de los responsables del área poseen un nivel de formación profesional y el 33\% no tienen ningún tipo de formación, es decir que existe un gran número de empresas que realizan empíricamente el mercadeo, dado esto la empresa considera al mercadeo como una área para incentivar las ventas, aunque no podemos desmerecer esta consideración es muy importante desatacar que no se puede dejar a un lado el análisis de la competitividad.

Las empresas señalan entre sus principales estrategias la realización de redes sociales, el buen servicio y descuentos por temporadas, estas estrategias por así llamarlas se las realiza sin un estudio previo o un plan son realizadas empíricamente los gerentes señalan que les funciona este tipo de acciones, pero no tienen los resultados siempre esperados.

Las empresas estudiadas se encuentran en general en la ciudad de Machala y el 59\% tienen más de 7 años en el mercado Orense y son de tipo independiente.

\section{Conclusiones}

En base a todos la bibliografía recabada y al análisis de los datos encuestados podemos concluir que si la provincia de El Oro quiere resaltar su nivel de competitividad es de clara relevancia para la situación económica que tiene que tener en cuenta implementar un plan de marketing estructurado, que no es más que una guía de ruta y seguir los lineamientos de este, como son ¿Dónde nos encontramos?, ¿hacia dónde queremos llegar?, y ¿Qué podemos hacer para llegar?; si la empresa no está en la capacidad de tener un área exclusivamente de mercadeo podemos sugerir que la empresa contrate una persona externa o empresa que le brinde los servicios externos del manejo del área de mercadeo y sus estrategias. 


\section{Bibliografía}

AMA. (2004). Dioctionary of Marketing Terms. Recuperado el 05 de septiembre de 2007, de Http://www.marketingpower.com/mg-dictionary-view1862.php?

Carson, D., \& Cromie, S. M. (2010). Marketing and entrepreneurship in SMEs. An innovative Approach.

Chirinos Cuadros, C. R., \& Rosado Samaniego, J. F. (2018). Estrategia de diferenciaciòn: el caso de las empresas industriales. Redalyc, 165-174.

Donrrosoro, I., Garcia C, Gonzalez, M., Lezamiz, M., Matey, J., Moso, M., y otros. (2001). El modelo de estión de las PYMESs vascas de éxito. Bilbao: PMP, Cluster del Conocimiento y ediciones.

George, Z. S. (2002). International entrepreneurship: the current status of the field and future research agenda. Strategic Entrepreneurship: Creating a New Mindset, 255-288.

Inec. (2012). www.ecuadorencifras.gob.ec. Obtenido de Instituto nacional de estadisticas y censos: www.ecuadorencifras.gob.ec

Kester, C., \& Luehrman, T. (1989). Are we feeling more competitive yet? The exchange rate gambit. Sloan Management Review, 19-22.

Kotler, P. (1995). Direccion de Mercadotecnia. Analisis, planeación, implementación y control. Madrid: Prentice Hall.

McClymont, H. \&. (2003). How to implement marketing streategies using database approaches. Database Marketing \& customer Strategy Management, 135-148.

Munuera, J. L. (2007). Estrategias de marketing: un enfoque basado en el proceso de dirección . ESIC.

Poutziouris, P. (2003). The strategic orientation of owner-managers of small ventures. International Journal of Entrepreneurial behaviour \& Reseach, 185-214.

Ramos, R. (2001). Modelos de evaluación de la competitividad Internacional: Una aplicación empirica al caso de las Islas Canarias. Tesis para obtener el grado de Doctor en Ciencias Economicas.

Srivastava, R. S. (1998). Market-based assets and shareholder value: a framework for analysis. Journal of Marketing, 2-18.

Villareal, R. (2006). El modelo de competitividad sistématica de los agro negocios en la cadena global de valor. IOSD y CECID.

Wensley, D. \&. (2010). Assesing advantage:A framework for diagnosis competitive superiority. Journal of marketing, 1-20.

Wind, Y. y. (1983). Marketing strategy: New directions for theory and research. Journal of Marketing, 1225. 